\title{
Motivaciones para el voluntariado: consideraciones teóricas y una propuesta de operacionalización
}

Motivations for volunteering: theoretical considerations and a proposal for operationalization

Motivações para o voluntariado: considerações teóricas e uma proposta de operacionalização

\section{María Carolina Klos ${ }^{1}$ Viviana Noemi Lemos ${ }^{2}$}

Recibido: 15 de noviembre de 2018

Aprobado: 20 de mayo de 2019

Publicado: 21 de junio de 2019

Cómo citar este artículo:

Klos, M. A. y Lemos, V. N. (2019). Motivaciones para el voluntariado: consideraciones teóricas y una propuesta de operacionalización. Pensando Psicología, 15(25), 1-29. DOI: https://doi.org/10.16925/2382-3984.2019.01.04

Artículo de investigación. https://doi.org/10.16925/2382-3984.2019.01.04

1 Universidad Adventista del Plata. Centro Interdisciplinario de Investigación en Psicología Matemática y Experimental Dr. Horacio J. A. Rimoldi (CIIPME)

ORCID: https://orcid.org/0000-0002-5108-4692

Correo electrónico: carolina.klos@uap.edu.ar

2 Universidad Adventista del Plata. Centro Interdisciplinario de Investigación en Psicología Matemática y Experimental Dr. Horacio J. A. Rimoldi (CIIPME)

ORCID: https://orcid.org/0000-0002-8855-2293 


\title{
Resumen
}

Introducción: El voluntariado puede definirse como conductas prosociales no espontáneas, planificadas a largo plazo dadas en un contexto organizacional y que buscan activamente beneficiar a extraños (Penner, 2002). Objetivo: Dado el interés por conocer aquellos factores que impulsan a una persona a involucrarse y permanecer en el servicio voluntario, se presentan las principales consideraciones teóricas referidas a las motivaciones para el voluntariado, proponiendo un instrumento para su operacionalización.

Método: Con la colaboración de cinco jueces expertos se construyó un instrumento de 62 reactivos con un formato de respuesta tipo Likert de 7 puntos. Participaron del estudio 320 adultos jóvenes, de entre 18 a 30 años $(M=22,34 ; D E=3,36)$.

Resultados: Se analizaron las propiedades psicométricas de la escala a partir de: a) el poder discriminativo de los ítems, b) la estructura subyacente de la prueba, y c) la consistencia interna de la escala. Los resultados obtenidos evidencian un funcionamiento psicométrico adecuado.

Conclusiones: El cuestionario de evaluación de motivaciones para el voluntariado podría ser una herramienta útil para el reclutamiento de voluntarios, la ubicación de candidatos en puestos apropiados y para el diseño de estrategias que favorezcan su permanencia.

Palabras clave: análisis factorial, evaluación, motivaciones, propiedades psicométricas, voluntariado.

\begin{abstract}
Introduction: Volunteering can be defined as non-spontaneous, planned long-term prosocial behavior provided in an organizational context and that actively seeks to benefit strangers (Penner, 2002).

Objective: Given the interest in knowing the factors that drive a person to get involved and remain in voluntary service, the main theoretical considerations related to the motivations for volunteering are presented, proposing an instrument for its operationalization.

Method: With the aid of five expert judges, an instrument consisting of 62 questions was built with the answer sheet adopting a 7-point Likert format. 320 young adults, aged 18 to 30 years $(M=22.34$; $S D=3.36)$ participated in the study.

Results: The psychometric properties of the scale were analyzed based on: a) the discriminative power of the items, b) the underlying structure of the test, and c) the internal consistency of the scale. The results obtained show adequate psychometric validity.

Conclusions: The questionnaire for the evaluation of motivational reasons for volunteering could be a useful tool for recruiting volunteers, placing candidates in appropriate positions and designing strategies that encourage them to continue volunteering.
\end{abstract}

Keywords: factor analysis, evaluation, motivation, psychometric properties, volunteering.

\section{Resumo}

Introdução: 0 voluntariado pode ser definido como comportamentos pró-sociais de longo prazo, não espontâneos e planejados, dados em um contexto organizacional e buscando ativamente beneficiar estranhos (Penner, 2002).

Objetivo: Dado o interesse em conhecer os fatores que levam uma pessoa a se envolver e permanecer no serviço voluntário, são apresentadas as principais considerações teóricas relacionadas às motivações para o voluntariado, propondo um instrumento para sua operacionalização. 
Método: Com a colaboração de cinco juízes especialistas, um instrumento de 62 reagentes foi construído com um formato de resposta Likert de 7 pontos. Participaram do estudo 320 adultos jovens, com idades entre $18 \mathrm{e}$ 30 anos $(M=22,34 ; D P=3,36)$.

Resultados: As propriedades psicométricas da escala foram analisadas com base em: a) poder discriminativo dos itens; b) estrutura subjacente do teste; ec) consistência interna da escala. Os resultados obtidos mostram funcionamento psicométrico adequado.

Conclusões: 0 questionário de avaliação da motivação para o voluntariado pode ser uma ferramenta útil para o recrutamento de voluntários, colocando os candidatos em posições apropriadas e elaborando estratégias que favoreçam sua permanência.

Palavras-chave: análise fatorial, avaliação, motivações, propriedades psicométricas, voluntariado.

\section{Introduction}

A lo largo de los años, la psicología social ha demostrado un amplio interés en aquellos comportamientos sociales que involucran sacrificios personales por brindar ayuda a otros individuos, particularmente extraños (Batson, 1991; Clary et al., 1998; Eisenberg y Fabes, 1990; Erasmus y Morey, 2016; Garipova, 2016; Pham, 2019). Dentro del espectro de las conductas prosociales, múltiples estudios han focalizado su atención en la evaluación de aquellas conductas de ayuda a corto plazo, con la posibilidad de proporcionar un solo acto relativamente breve de ayuda y que tienen lugar en un ámbito doméstico (Clary et al., 1998; Parboteeah, Cullen, y Lim, 2004; Penner y Finkelstein, 1998).

La literatura académica, como así también los marcos jurídicos nacionales, exponen diversas definiciones sobre voluntariado. De acuerdo a la definición adoptada en la Asamblea General de las Naciones Unidas en 2001, la acción voluntaria debe cumplir requisitos como: 1) Ilevarse a cabo libremente, no como una obligación impuesta por una ley, contrato o requisito académico; 2) no debe realizarse para obtener una recompensa económica; 3) la acción debe beneficiar a otras personas o comunidad. Por lo tanto, las tres condiciones serían voluntad libre, motivación no monetaria y beneficio para otros. Se puede incluir como cuarta característica el carácter organizativo de la actividad (VNU, 2011). Existe cierto consenso para definir el voluntariado como conductas prosociales planificadas a largo plazo, que buscan de manera activa beneficiar a extraños y que se dan en un entorno organizacional (Erasmus y Morey, 2016; Penner, 2002). Por lo tanto, su estudio y evaluación difiere al de las conductas de ayuda desde su abordaje tradicional, es decir, como una respuesta ante situaciones específicas (Clary et al., 1998).

El fenómeno del voluntariado es una opción formal, pública y proactiva que implica proporcionar de manera voluntaria recursos personales, tales como el tiempo y esfuerzo, para promover el bienestar de otra persona, grupo u organización 
(Clary et al., 1998; Erasmus y Morey, 2016; Shye, 2010). El trabajo voluntario no es remunerado y se brinda a quienes el voluntario no debe obligaciones contractuales, familiares o de amistad (Parboteeah, Cullen, y Lim, 2004). A fin de sintetizar y unificar los múltiples postulados teóricos que se dan al voluntariado, Smith (2009) propone cinco dimensiones para su definición: 1) retribución económica: los voluntarios no deben recibir recompensa económica; 2) libre voluntad: el ejercicio debiera ser libre de obligación legal o contractual; 3) naturaleza del beneficio: la existencia de un beneficiario distinto al voluntario, es decir, el voluntario no debiera ser el único en obtener beneficios; 4) entorno organizacional: la actividad debe ser desarrollada desde una organización formal o informal; y 5) nivel de compromiso: un formato de la actividad periódico y a largo plazo.

Como se ha mencionado, una de las características del voluntariado es la ausencia de compensación monetaria, sin embargo, diversos estudios han demostrado que los voluntarios pueden esperar otro tipo de recompensas (Gage y Thapa, 2012). Por lo tanto, el voluntariado podría resultar en un beneficio tanto para los destinatarios del servicio como para los voluntarios (Gage y Thapa, 2012).

El voluntariado también deriva en beneficios sociocomunitarios ya que las actividades de ayuda y otras conductas prosociales promueven la armonía social. Tanto es así, que gran parte de las organizaciones sin fines de lucro y no gubernamentales del mundo, pueden existir y ser efectivas debido a la participación de voluntarios (Dutta-Bergman, 2004; Hustinx, et al., 2010; Stukas, Hoye, Nicholson, Brown, y Aisbett, 2016). Por lo tanto, el voluntariado da respuesta a múltiples necesidades sociales y ambientales, representando así una pieza fundamental para la sostenibilidad de la sociedad civil en el mundo (Hustinx, et al., 2010).

Por esta razón, mediante diversos estudios se ha procurado identificar al "voluntario prototípico" o indagar acerca del por qué algunas personas se interesan por actividades de voluntariado mientras otras no (Curtis, Grabb, y Baer 1992; Hettman y Jenkins 1990; Pham, 2019; Thoits y Hewitt; Wilson y Musick 1997). Penner (2002) plantea que, aparte de las características de la situación que demanda ayuda, los factores más influyentes en la decisión de ser voluntario son los aspectos demográficos -edad, ingresos, y nivel de educación-, predisposicionales y las características de la organización. Sin embargo, debido a que el proceso es interaccional y muy dinámico, estos factores no proporcionan una explicación absoluta y completa de por qué las personas deciden ser voluntarias y mantienen una continuidad en un período extendido de tiempo.

Los factores que predisponen al voluntariado son atributos relativamente duraderos de los individuos, tales como las creencias religiosas, los valores personales, 
los rasgos de personalidad y las motivaciones que subyacen al voluntariado. Estos factores tienen una influencia directa tanto en la posibilidad de que la persona considere participar en actividades de voluntariado como en la decisión de ofrecerse como voluntario (Penner, 2002).

Para sintetizar los aspectos que predisponen al voluntariado, Thoits y Hewitt (2001) plantean cuatro modelos explicativos que persiguen integrar los aportes realizados en el área. El primer modelo se denomina "modelo de valores y actitudes". Se señala como un segundo modelo el "modelo de identidad de rol" o "modelo de identidad grupal", el tercer modelo se denomina, "modelo de personalidad voluntaria" y el cuarto modelo, "modelo de motivaciones voluntarias", donde se enfatiza en las motivaciones de voluntariado y se incluyen las diversas metas que se persiguen al involucrarse en esta actividad -aprendizaje de nuevas habilidades, desarrollo personal, autoestima, compromiso, entre otras-, lo que implica que el voluntariado puede servir a diferentes fines (Penner y Finkelstein 1998; Penner, Midili, y Kegelmeyer, 1997). Thoits y Hewitt (2001) sugieren la presencia de un quinto modelo explicativo, "el modelo de bienestar personal", que examina la personalidad, la salud física y la salud mental como aspectos que disponen al voluntariado.

La presente investigación se centra en el cuarto modelo, el modelo de las motivaciones voluntarias; el abordaje de los modelos restantes excede al presente artículo. Por lo tanto, se describen las principales teorías explicativas sobre motivaciones ligadas al voluntariado y las herramientas de evaluación más estudiadas.

\section{Conceptualización de las motivaciones para el voluntariado}

Dado que las actividades de voluntariado involucran un tipo de ayuda sostenida y continua, diversos estudios han procurado conocer las motivaciones subyacentes, es decir, aquellos procesos que mueven a las personas a la acción y que, por lo tanto, la inician, dirigen y sostienen (Bang y Ross, 2009; Burns et al., 2005; Carlo, Okun, Knight, y Guzman, 2005; Clary et al., 1998; Hustinx, et al., 2010; Parboteeah, Cullen, y Lim, 2004; Pham, 2019).

Una comprensión más profunda y un conocimiento más específico acerca de las motivaciones para el voluntariado contribuyen al reclutamiento y ubicación de candidatos en puestos apropiados (Allison, Okun, y Dutridge, 2002; Shye, 2010). A su vez, permiten incentivar a los voluntarios a que participen y permanezcan involucrados en las organizaciones que brindan ayuda, lo cual posibilita el cumplimiento de objetivos institucionales que persiguen beneficiar a otras personas o al medio ambiente 
y, por lo tanto, tienen una relevancia para la comunidad al garantizar la prestación de servicios sociales de mayor calidad (Butt, Hou, Soomro, y Acquadro Maran, 2017; Hibbert, Piacentini, y Dajani, 2003).

La literatura científica respecto a las motivaciones para el voluntariado se puede dividir en modelos, de acuerdo a la cantidad de motivaciones que se incluyen en su estudio (Butt, Hou, Soomro, y Acquadro Maran, 2017). El modelo de Cnaan y GoldbergClen (1999) explica la motivación para el voluntariado a nivel teórico y empírico desde una concepción unidimensional. Así también, puede incluirse como modelo de único factor la teoría de la responsabilidad unificada de Dutta-Bergman (2004) en la que se establece que el hilo conductor que subyace a la motivación para el voluntariado es la responsabilidad personal, es decir, se expone la noción de que quienes llevan una vida responsable consigo mismos, también ejercen responsabilidad mediante el voluntariado, con el fin de brindar ayuda a los demás (Dutta-Bergman, 2004).

Las teorías de más de un factor para explicar las motivaciones para el voluntariado surgieron con la introducción del concepto de altruismo, de esta forma, comenzaron los estudios de motivaciones altruistas y no altruistas (Batson y Ahmad, 2001). Uno de los primeros modelos existentes divide a las motivaciones para el voluntariado en aquellas ligadas a la preocupación por el bienestar de los demás -motivaciones altruistas - y aquellas ligadas al propio interés o al sentirse bien con uno mismo -motivaciones egoístas - (Frisch y Gerard, 1981; Geiser, Okun, y Grano, 2014; Latting, 1990; Shye, 2010; Smith, 1981). Otra de las teorías bifactoriales establece que es posible estudiar motivaciones para el voluntariado de carácter intrínseco y extrínseco (Gidron, 1985; Geiser, Okun, y Grano, 2014). Siendo las motivaciones extrínsecas las que responden a incentivos externos y las intrínsecas a motivos de valor, interés y disfrute de la actividad (Geiser, Okun, y Grano, 2014). El debate resultante de la dualidad altruismo-egoísmo ha llevado a dos argumentos opuestos, por un lado, la idea de que toda actividad de ayuda voluntaria se encuentra motivada por un deseo egoísta de obtener beneficio para uno mismo y, por el otro, la idea de que la ayuda voluntaria se basa en una preocupación por los demás, carente de intereses egoístas (Clary y Snyder, 1999).

El modelo de tres factores para explicar las motivaciones para el voluntariado adicionó a las motivaciones altruistas y egoístas, la motivación social (Fitch, 1987; Butt, Hou, Soomro, y Acquadro Maran, 2017). Mientras que otro modelo tripartito estableció que es posible diferenciarlas en motivaciones altruistas, sociales y materiales - como por ejemplo obtener o potenciar ciertas habilidades- (Butt, Hou, Soomro, y Acquadro Maran, 2017). 
Como se ha mencionado, las acciones que involucran las actividades de voluntariado son diversas, complejas y sostenidas en el tiempo, por lo que es factible que las motivaciones que poseen las personas para involucrarse y permanecer a largo plazo como voluntarios sean multifacéticas. Se ha observado que no es apropiado clasificar las motivaciones de voluntariado en polos altruistas o egoístas, ya que algunos motivos que poseen los voluntarios combinan intereses por los demás y por uno mismo (Clary y Snyder, 1999). Si bien el altruismo se reporta comúnmente como la principal motivación para ser voluntario, la investigación contemporánea tiende a descartar motivos puramente idealistas para así investigar respecto a ventajas personales que pueden impulsar a los voluntarios. Mientras que los motivos relacionados con beneficiar a los demás llevarían a las personas a involucrarse en la actividad voluntaria, otras motivaciones serían más relevantes para la participación continua (Gage y Thapa, 2012).

Debido a la existencia de múltiples factores motivacionales que impulsan a una persona a que se involucre y permanezca activa en el servicio voluntario, Clary et al. (1998) han desarrollado y operacionalizado un modelo más complejo, compuesto por seis factores, basado en el enfoque funcional de la psicología social. El núcleo central de la teoría funcional es que las personas pueden participar en la misma actividad, pero tener distintos motivos subyacentes para hacerlo (Clary y Snyder, 1991). Por lo tanto, desde la teoría funcional, las personas pueden ser voluntarias persiguiendo distintos objetivos, y el mismo voluntario puede tener más de un motivo para hacerlo (Clary y Snyder, 1999).

Los seis factores que conforman la teoría de Clary et al. (1998) son los siguientes: 1) valores: oportunidades para expresar los valores propios asociados a preocupaciones altruistas y de servicio a los demás; 2) comprensión: oportunidades para experimentar, aprender y ejercitar los propios conocimientos; 3) social: oportunidad de estar con amigos y de realizar una actividad valorada socialmente; 4) carrera: contribución a la formación profesional o ejercitar habilidades ligadas a la carrera; 5) protección: a fin de abordar problemas personales o para reducir la culpa por encontrarse en una situación más favorable que otros; y 6) mejora: oportunidad para mantener o mejorar el afecto positivo, relacionado con el crecimiento personal y autoestima (Clary et al.,1998).

La mayoría de las investigaciones que abordan el estudio de las motivaciones de voluntariado lo hacen desde la teoría de los seis factores de Clary et al. (1998), ya que ha tenido un alto apoyo teórico y respaldo empírico (Gage y Thapa, 2012; Stukas, Daly y Clary, 2006). Al estudiar las motivaciones en categorías o funciones, se pueden alcanzar niveles más altos de especificidad respecto a las motivaciones que poseen 
determinados grupos o individuos, y además posibilita conocer qué tipos de motivaciones son las que prevalecen (Clary y Snyder, 1999; Gage y Thapa, 2012).

\section{Evaluación de las motivaciones para el voluntariado}

La complejidad conceptual de las motivaciones para el voluntariado se ve reflejada en la metodología de su evaluación (Clary et al., 1998). Clary et al. (1998) han creado un inventario para la operacionalización de las motivaciones para el voluntariado desde la teoría que postulan. El Inventario de Funciones del Voluntariado (VFI; Volunteer Function Inventory, Clary et al., 1998) ha sido adaptado a distintos países y utilizado en diversas investigaciones (Bierhoff, Schu, Iken, y Hoof, 2007; Hallmann y Harms, 2012; Jiranek, Kals, Humm, Strubel, y Wehner, 2013; Rokach y Wanklyn, 2009; Van Vianen, Nijstad, y Voskuijl, 2008). El instrumento evalúa mediante una escala Likert (1 a 7 ) las respuestas que dan los individuos a 30 razones para ser voluntario (5 por cada dimensión). Se les pide que reporten en el autoinforme la importancia que tiene para ellos cada uno de los motivos de voluntariado (Allison, Okun, y Dutridge, 2002).

Si bien los resultados derivados de investigaciones previas respaldan el uso del VFI para evaluar motivos de voluntariado por la facilidad de su administración y por sus propiedades psicométricas satisfactorias y consistentes con las expectativas teóricas, gran parte de los investigadores se ha encontrado con ciertas dificultades en la adaptación del instrumento, por lo que ha tenido que enmendar o agregar otros motivos (Allison, Okun, y Dutridge, 2002; Butt, Hou, Soomro, y Acquadro Maran, 2017). Esta situación ha resultado en variaciones sustanciales del instrumento y distintas versiones del mismo, especialmente cuando se aplica en algún contexto específico (Erasmus y Morey, 2016; Butt, Hou, Soomro, y Acquadro Maran, 2017).

Por lo tanto, fueron incluidas en las nuevas versiones del inventario dimensiones como religiosidad, disfrute y formación de equipos (Allison, Okun, y Dutridge, 2002). Así también, en la versión australiana se adicionaron las dimensiones de religiosidad, factores sociales y gubernamentales (Bierhoff, Schu, Iken y Hoof, 2007). Van Vianen, Nijstad y Voskuijl (2008) adicionaron la dimensión "pasatiempo", quedando la escala conformada por siete factores. En el estudio de Rokach y Wanklyn (2009) también agregaron una dimensión a la que llamaron "disfrute". Jiranek, Kals, Humm, Strubel y Wehner (2013) añadieron la dimensión de "justicia social". Otros autores han realizado adaptaciones para adecuar el cuestionario a contextos específicos como el ámbito de voluntariado deportivo (Hallmann y Harms, 2012).

Las evaluaciones de las motivaciones de voluntariado mediante cuestionarios cerrados posibilitan obtener puntuaciones de las distintas dimensiones y facilitan 
la valoración de las mismas. Sin embargo, presentan limitaciones como la elevada deseabilidad y la posible confusión de los evaluados entre motivos y expectativas (Chacón, Pérez, Flores, y Vecina, 2010). Por esta razón, otra forma de estudiar las motivaciones para el voluntariado es mediante preguntas abiertas. Si bien este tipo de investigaciones son más costosas por el tiempo que requieren, han resultado ser grandes aportes, de carácter cualitativo, para conocer más acerca de las motivaciones que poseen los voluntarios (Chacón, et al., 2010).

El estudio de Chacón y colaboradores (2010) se llevó a cabo en España mediante la evaluación de 1515 voluntarios pertenecientes a 132 organizaciones, se realizó la evaluación de las motivaciones para el voluntariado mediante una pregunta abierta. La categorización de las 4017 respuestas proporcionadas por los voluntarios fue realizada teniendo en cuenta la teoría y dimensiones que se contempla en el VFI $y$, a la vez, se incluyeron las motivaciones que adicionaron otros autores: interés por la comunidad, relaciones sociales, valores religiosos, disfrute y trabajo en equipo. Por lo tanto, fueron definidas 11 categorías. De otro lado, las mismas fueron redefinas por los jueces al ubicar las respuestas, por lo que se adicionaron las categorías de interés en la actividad y compromiso organizacional. Además, dentro de las categorías originales se identificaron subcategorías (Chacón, et al., 2010).

En este contexto, teniendo en cuenta las categorías y subcategorías de motivaciones para el voluntariado planteadas por Chacón et al. (2010), se desprende el presente artículo que tiene por objetivo presentar una propuesta de operacionalización de las motivaciones para el voluntariado dirigida a adultos jóvenes y explorar preliminarmente su funcionamiento psicométrico.

\section{Método}

\section{Diseño del instrumento}

De acuerdo a los objetivos planteados y a los análisis estadísticos utilizados para su consecución, la presente investigación de tipo cuantitativo se desarrolló mediante un diseño de estudio instrumental (Gibbons, Flores y Mónico, 2004; Montero y León, 2007; Servera y Cardo, 2006).

\section{Participantes}

Los participantes fueron evaluados en un evento de carácter internacional realizado en el año 2017 en una universidad privada de Argentina, en la provincia de Entre Ríos, 
dirigido a interesados en actividades de voluntariado, por lo que la muestra inicial estuvo conformada por individuos de diversas nacionalidades y edades. Para seleccionar a los participantes que finalmente conformarían la muestra de estudio, se aplicó como criterio de inclusión que los participantes estuvieran participando de manera activa en alguna organización de voluntarios, que sean residentes de Argentina y que tuvieran entre 18 y 30 años. La muestra inicial superó los 500 participantes, pero tras aplicar los criterios de inclusión la cantidad se redujo.

Se entregó a cada participante un consentimiento informado que explicaba el trabajo en cuestión y exponía garantías de confidencialidad de los datos proporcionados, siguiendo los lineamientos de la Ley Nacional 25 326. Los individuos que firmaron el acuerdo accedieron a completar el cuestionario.

Se trabajó con una muestra no probabilística intencional, conformada por 320 participantes de 18 a 30 años $(M=22,34 ; D E=3,36)$, de los cuales 203 fueron mujeres (63,4 \%) y 117 hombres (36,6 \%). De acuerdo a la información recabada mediante un cuestionario sociodemográfico, del total de la muestra, 213 personas (66,6 \%) habían estado involucradas en el pasado en actividades de voluntariado, por lo tanto, con experiencia en el desempeño como voluntarios y 107 (33,4 \%) habían iniciado ese año.

La muestra de terapeutas presentó una experiencia clínica promedio de 16,16 años $(D E=13,40)$, siendo el $67 \%$ de los participantes de género femenino. De los 101 terapeutas que participaron en el estudio, 49 se autoproclamaron psicoanalíticos-psicodinámicos, 30 cognitivo-conductuales, 20 cognitivo-integrativos y 2 psicoanalítico-integrativos. El 57,4 \% de las sesiones puntuadas correspondían a pacientes mujeres y la edad media de la muestra de pacientes fue de 36,27 años ( $D E=10,78)$. E $15,8 \%$ se hallaban en el inicio del tratamiento, el 76,2 \% en un momento intermedio y el 7,9 \% finalizando el mismo. El diagnóstico de los pacientes se estableció a partir del reporte de los terapeutas. Los trastornos más prevalentes en la muestra fueron los trastornos de ansiedad (26,7 \%), los trastornos del estado de ánimo (17,8 \%) y los trastornos de personalidad (13,9\%). Se detallaron, además, diagnósticos de neurosis $(9,9 \%)$, trastorno borderline (9,9\%), crisis vitales/de desarrollo personal y otros $(18,8 \%)^{1}$.

\section{Instrumentos y procedimientos}

El Cuestionario Argentino de Motivaciones para el Voluntariado (CAMV) fue diseñado y administrado a los fines de evaluar las motivaciones para el voluntariado presentes en adultos jóvenes argentinos.

1 Los diagnósticos reportados corresponden a distintos sistemas nosográficos debido a que fueron aportados espontáneamente por los terapeutas, sin presentarles opciones de respuesta. 


\section{Procedimientos seguidos para la construcción del instrumento}

Las contribuciones derivadas del estudio cualitativo de Chacón et al. (2010), quienes proponen la evaluación de las motivaciones para el voluntariado mediante una pregunta abierta, han servido como plataforma para la construcción del CAMv. En su investigación, las respuestas proporcionadas por los participantes se clasifican en una serie de categorías que tienen su fundamento teórico en el enfoque funcional de las motivaciones de voluntariado propuesto por Clary et al. (1998) y que a su vez adiciona los aportes de otros investigadores del área (Allison, Okun, y Dutrige, 2002; Chacón y Vecina, 1999; Omoto y Snyder, 1995).

Clary et al. (1998) establecen las dimensiones: valores, comprensión, ajuste social, mejora del currículum, defensa del yo y mejora de la estima. Estas seis dimensiones son evaluadas mediante el instrumento VFI (Clary et al., 1998). La motivación de interés por la comunidad se adiciona del estudio de Omoto y Snyder (1995) la cual es evaluada por medio de cMvs (Omoto y Snyder, 1995). De la adaptación al español del CMVs, se incluyó la motivación basada en relaciones sociales (Chacón y Vecina, 1999). Las dimensiones valores religiosos, disfrute y trabajo en equipo, se desprenden de los aportes de Allison, Okun y Dutrige (2002).

Por lo tanto, las categorías construidas a partir de los estudios previos de las motivaciones de voluntariado y del estudio de Chacón, et al. (2010) quedaron conformadas como se expone en la tabla 1.

Tabla 1. Categorías y subcategorías de las motivaciones para el voluntariado propuestas por Chacón et al. (2010)

\begin{tabular}{ll}
\hline \multicolumn{1}{c}{ Categoría de Motivaciones } & \multicolumn{1}{c}{ Subcategorías } \\
\hline 1) Valores & 1.1) Valores religiosos \\
& 1.2) Valores de transformación social \\
& 1.3) Valores de reciprocidad \\
& 1.4) Valores de interés por la comunidad \\
& 1.4.1) Valores de ayuda a un territorio específico. \\
& 1.4.2) Valores de ayuda a un colectivo específico. \\
\hline 2) Conocimiento-comprensión & 2.1) Conocimiento de otros \\
\hline 3) Ajuste social & 2.2) Auto-conocimiento \\
\hline 4) Mejora del currículum & \\
\hline 5) Defensa del Yo & \\
\hline
\end{tabular}




\begin{tabular}{ll}
\hline \multicolumn{1}{c}{ Categoría de Motivaciones } & \multicolumn{1}{c}{ Subcategorías } \\
\hline 6) Mejora de la estima & 6.1) Estima \\
& 6.2) Crecimiento personal \\
& 6.3) Relaciones sociales \\
& 6.4) Disfrutar \\
\hline 7) Compromiso organizacional & 7.1) Compromiso institucional \\
\hline 8) Interés en la actividad & 7.2) Compromiso con el grupo \\
\hline 9) Condicionales & 8.1) Interés en la actividad específica \\
\hline 10) Otros & \\
\hline
\end{tabular}

Fuente: elaboración propia

En base a las categorías y subcategorías expuestas con anterioridad y a las definiciones y ejemplos de las razones dadas por los voluntarios en cada grupo, se redactó un conjunto inicial de 74 ítems que contemplaban cada una de las dimensiones -entre tres y cinco reactivos por categoría y subcategoría-. A continuación, se contactó a cinco jueces expertos en el área de evaluación psicológica y psicometría para que evaluaran: 1) si los ítems reflejaban la dimensión y subdimensión a evaluar -teniendo en cuenta las definiciones teóricas-; 2) si la redacción de los ítems era clara, teniendo presente que la población de estudio tenía de 18 años en adelante; y 3) si consideraban más apropiada una redacción alternativa para expresar la idea del enunciado. También fue dada la posibilidad de proponer nuevos ítems.

De acuerdo a las consideraciones de los jueces, se realizaron correcciones en cuanto a expresiones y se descartaron algunos ítems considerados redundantes. En esta etapa la escala a estudiar quedó conformada por 62 enunciados, representando las categorías y subcategorías: valores, conocimiento, ajuste social, mejora de CV, mejora de estima y condicionales. Las dimensiones: otros, interés en la actividad, defensa del yo y compromiso organizacional no fueron contempladas porque, de acuerdo a los criterios de los jueces expertos, los ítems resultaban reiterativos o se solapaban con los ítems de otras dimensiones. El formato de respuesta escogido fue tipo Likert, donde los sujetos debieron indicar en una escala del 1 -no coincide en nada con mis motivos - al 7 -coincide fuertemente con mis motivos- su grado de identificación con el enunciado.

Algunos ejemplos de los reactivos de las categorías son los siguientes: 1) valores: "asumir mi responsabilidad religiosa"; 2) conocimiento-comprensión: "vivir otras realidades"; 3) ajuste social: "compartir momentos con amigos que también se 
involucran"; 4) mejora del currículum: "desarrollar habilidades relacionadas con mi profesión"; 5) mejora de la estima: "para sentirme bien conmigo mismo"; y 6) condicionales: "ocupar mis vacaciones en algo productivo".

La propuesta del CAMV, conformado por 62 ítems, fue administrado inicialmente a un grupo reducido de adultos jóvenes argentinos a fin de corroborar la comprensión de los enunciados y conocer el tiempo estimado de su administración -tiempo de resolución promedio: 15 minutos-. A su vez, la escala dispone de un espacio para que los participantes puedan responder libremente cuál es su principal motivación para realizar actividades de voluntariado.

\section{Procedimientos para el análisis de datos}

Se siguieron las normas para el desarrollo de estudios instrumentales expuestas por Carretero-Dios y Pérez (2005), las cuales incluyen: a) justificación del estudio; b) delimitación conceptual del constructo a evaluar; c) construcción y evaluación cualitativa de los ítems; d) estudio de la dimensionalidad del instrumento; e) estimación de la fiabilidad; y f) estudio de evidencias externas de validez. Los puntos a y b fueron abordados en el apartado "introducción". Dado que en el presente estudio se persigue presentar una propuesta inicial de operacionalización de las motivaciones para el voluntariado, queda reservado para un estudio posterior el estudio de evidencias externas de validez y la confirmación de la estructura propuesta en este estudio, a través de un Análisis Factorial Confirmatorio.

Primero, se calculó el poder discriminativo de los ítems mediante prueba $U$ de Mann-Whitney. Luego, se seleccionaron los "mejores" ítems que definieran una clara estructura subyacente de la prueba a través de múltiples Análisis Factoriales Exploratorios (AFE) y, por último, se evaluó la consistencia interna de la escala por medio de los coeficientes de alpha de Cronbach y Omega de McDonald.

\section{Resultados}

\section{Análisis del poder discriminativo}

Se realizó el análisis del poder discriminativo de los ítems mediante el estadístico no paramétrico $U$ de Mann-Whitney, dado que la prueba de Kolmogorov-Smirnov indicó ausencia de normalidad en la distribución de los mismos. Los resultados indican que todos los ítems del instrumento resultaron significativos $(p<0,001)$ respecto a las diferencias existentes entre los rangos ubicados por debajo del percentil 25 y los 
ubicados por encima del percentil 75. Por lo tanto, se concluye que los 62 reactivos resultan discriminativos.

Tabla 2. Dimensión Valores (subdimensiones: religiosos, transformación social, reciprocidad, interés por la comunidad)

\begin{tabular}{ccccc}
\hline Reactivo & Grupo inferior & Grupo superior & & \\
\hline & Cuartil inferior & Cuartil superior & $\boldsymbol{U}$ & 0,000 \\
\hline 1 & 66,42 & 103,70 & 2027,00 & 0,000 \\
\hline 12 & 67,12 & 103,3 & 2085,00 & 0,000 \\
\hline 40 & 54,98 & 114,61 & 1077,50 & 0,000 \\
\hline 21 & 54,69 & 114,89 & 1053,50 & 0,000 \\
\hline 25 & 52,98 & 116,53 & 911,00 & 0,000 \\
\hline 33 & 54,49 & 115,09 & 1036,50 & 0,000 \\
\hline 36 & 51,14 & 118,28 & 758,50 & 0,000 \\
\hline 16 & 49,11 & 120,22 & 590,00 & 0,000 \\
\hline 26 & 54,71 & 114,87 & 1055,00 & 0,000 \\
\hline 49 & 50,78 & 118,62 & 729,00 & 0,000 \\
\hline 50 & 50,24 & 119,14 & 684,00 & 0,000 \\
\hline 60 & 49,13 & 120,20 & 592,00 & 0,000 \\
\hline 2 & 54,87 & 114,72 & 1068,50 & 0,000 \\
\hline 31 & 62,51 & 107,44 & 1702,00 & 0,000 \\
\hline 46 & 51,78 & 117,67 & 811,50 & 0,000 \\
\hline 61 & 51,43 & 118,01 & 1053,5000 \\
\hline 7 & 54,69 & 114,89 & 110,47 & 0,000 \\
\hline
\end{tabular}

Fuente: elaboración propia

Tabla 3. Dimensión Conocimiento (subdimensiones: conocimiento de otros, autoconocimiento)

\begin{tabular}{ccccc}
\hline Reactivo & Grupo inferior & Grupo superior & & \\
\hline & Cuartil inferior & Cuartil superior & $\boldsymbol{U}$ & $\boldsymbol{p}$ \\
\hline 11 & 51,69 & 113,98 & 704,50 & 0,000 \\
\hline 12 & 69,03 & 93,82 & 2196,00 & 0,000 \\
\hline 20 & 50,67 & 115,16 & 617,00 & 0,000 \\
\hline 38 & 46,59 & 119,91 & 266,00 & 0,000 \\
\hline 47 & 49,62 & 116,39 & 526,00 & 0,000 \\
\hline
\end{tabular}


(viene)

\begin{tabular}{ccccc}
\hline Reactivo & Grupo inferior & Grupo superior & & \\
\hline & Cuartil inferior & Cuartil superior & $\boldsymbol{U}$ & $\boldsymbol{p}$ \\
\hline 51 & 50,40 & 115,48 & 593,50 & 0,000 \\
\hline 24 & 46,52 & 119,99 & 260,00 & 0,000 \\
\hline 32 & 46,27 & 120,51 & 238,50 & 0,000 \\
\hline 43 & 46,07 & 120,51 & 221,00 & 0,000 \\
\hline 55 & 46,60 & 119,89 & 267,00 & 0,000 \\
\hline 8 & 50,83 & 114,98 & 630,50 & 0,000 \\
\hline
\end{tabular}

Fuente: elaboración propia

Tabla 4. Dimensión Ajuste Social (unidimensional)

\begin{tabular}{ccccc}
\hline Reactivo & Grupo inferior & Grupo superior & & \\
\hline & Cuartil inferior & Cuartil superior & $\boldsymbol{U}$ & $\boldsymbol{p}$ \\
\hline 19 & 53,02 & 120,79 & 599,50 & 0,000 \\
\hline 30 & 48,18 & 126,88 & 154,50 & 0,000 \\
\hline 42 & 48,90 & 125,97 & 221,00 & 0,000 \\
\hline 54 & 53,21 & 120,55 & 617,00 & 0,000 \\
\hline 44 & 47,32 & 127,97 & 75,00 & 0,000 \\
\hline
\end{tabular}

Fuente: elaboración propia

Tabla 5 . Dimensión Mejora de CV (unidimensional)

\begin{tabular}{ccccc}
\hline Reactivo & Grupo inferior & Grupo superior & & \\
\hline & Cuartil inferior & Cuartil superior & $\boldsymbol{U}$ & $\boldsymbol{p}$ \\
\hline 14 & 53,68 & 112,87 & 907,50 & 0,000 \\
\hline 27 & 44,59 & 122,76 & 135,50 & 0,000 \\
\hline 35 & 44,32 & 123,06 & 112,50 & 0,000 \\
\hline 59 & 43,16 & 124,32 & 14,00 & 0,000 \\
\hline
\end{tabular}

Fuente: elaboración propia 
Tabla 6. Dimensión Estima (subdimensiones: estima, crecimiento personal, relaciones sociales, disfrutar)

\begin{tabular}{|c|c|c|c|c|}
\hline Reactivo & Grupo inferior & Grupo superior & & \\
\hline & Cuartil inferior & Cuartil superior & $\boldsymbol{U}$ & $p$ \\
\hline 45 & 46,69 & 113,44 & 460,50 & 0,000 \\
\hline 28 & 45,10 & 115,13 & 332,00 & 0,000 \\
\hline 5 & 53,48 & 106,20 & 1011,00 & 0,000 \\
\hline 17 & 45,31 & 114,90 & 349,50 & 0,000 \\
\hline 53 & 52,80 & 106,93 & 955,50 & 0,000 \\
\hline 62 & 52,30 & 107,45 & 915,50 & 0,000 \\
\hline 56 & 52,73 & 107,00 & 950,00 & 0,000 \\
\hline 48 & 54,20 & 105,43 & 1069,50 & 0,000 \\
\hline 22 & 48,56 & 111,45 & 612,00 & 0,000 \\
\hline 10 & 57,05 & 102,39 & 1300,00 & 0,000 \\
\hline 6 & 49,65 & 110,28 & 701,00 & 0,000 \\
\hline 9 & 44,01 & 116,30 & 243,50 & 0,000 \\
\hline 23 & 44,40 & 115,88 & 275,50 & 0,000 \\
\hline 58 & 42,49 & 117,91 & 120,50 & 0,000 \\
\hline 3 & 69,13 & 89,52 & 2278,50 & 0,001 \\
\hline 18 & 46,17 & 113,99 & 419,00 & 0,000 \\
\hline 37 & 62,40 & 96,69 & 1733,50 & 0,000 \\
\hline 41 & 47,46 & 112,62 & 523,00 & 0,000 \\
\hline
\end{tabular}

Fuente: elaboración propia

Tabla 7. Dimensión Condicionales (unidimensional)

\begin{tabular}{ccccc}
\hline Reactivo & Grupo inferior & Grupo superior & & \\
& Cuartil inferior & Cuartil superior & $\boldsymbol{U}$ & $\boldsymbol{p}$ \\
\hline 4 & 55,79 & 114,80 & 817,50 & 0,000 \\
\hline 13 & 51,53 & 120,72 & 421,00 & 0,000 \\
\hline 34 & 50,51 & 122,13 & 326,50 & 0,000 \\
\hline 59 & 48,29 & 125,34 & 111,50 & 0,000 \\
\hline
\end{tabular}

Fuente: elaboración propia

\section{Análisis de la estructura subyacente del instrumento}

En primer lugar, se realizaron análisis factoriales exploratorios (AFE), por cada grupo de motivaciones, o dimensiones que conforman el instrumento. En todos los casos se utilizó el método de Máxima Verosimilitud (ML), por considerarse las variables 
continuas - poseen siete opciones de respuesta- y presentar valores de asimetría y curtosis menores o próximos a los guarismos de \pm 2 (Lloret-Segura, Ferreres-Traver, Hernández-Baeza, y Tomás-Marco, 2014). A su vez, se utilizaron rotaciones oblicuas (Oblimin) dado que las correlaciones entre algunos factores resultaron superiores a 32 (Tornimbeni, Pérez, y Olaz, 2008), tal como puede observarse en la matriz de correlaciones factoriales (ver tabla 11).

Los AFE posibilitaron la depuración de ítems del cuestionario permitiendo seleccionar aquellos que pesaron con buena carga en el factor, excluyendo, en términos generales, a aquellos con pesaje inferior a .50 o que presentaran complejidad factorial o carga en factor residual. Estos análisis también permitieron evaluar, si las subdimensiones encontradas empíricamente y los ítems que las operacionalizaban se correspondían con las categorías teóricas presentadas en la tabla 1 utilizada para la formulación de los mismos.

En la siguiente tabla se presentan de forma sintetizada los ítems que se conservaron y se descartaron de cada dimensión, de acuerdo a los criterios mencionados con anterioridad. A su vez, se muestran las reagrupaciones que se observaron de los subdimensiones.

Tabla 8. Tabla que sintetiza agrupación teórica y empírica de los ítems, luego de aplicar AFE.

\begin{tabular}{|c|c|c|c|}
\hline Dimensión & Agrupación teórica & $\begin{array}{l}\text { Agrupación empírica } \\
\text { AFE }\end{array}$ & Ítems eliminados \\
\hline \multirow[t]{4}{*}{ Valores } & Religiosos: $1,12,29$ y 40 & F1: $49,50,60$ y 26 & Inferior .50: 16, 61 \\
\hline & $\begin{array}{l}\text { Transformación social: } 21 \text {, } \\
25,33 \text { y } 36\end{array}$ & $\mathbf{F} 2: 25,21,63$ y 36 & $\begin{array}{l}\text { Complejidad factorial: } 46 \text {, } \\
31,33,29,40\end{array}$ \\
\hline & $\begin{array}{l}\text { Reciprocidad: } 16,26,49 \\
50 \text { y } 60\end{array}$ & F3: 1,2 y 12 & \\
\hline & $\begin{array}{l}\text { Interés por la comunidad: } \\
2,31,46,61 \text { y } 7\end{array}$ & & \\
\hline \multirow[t]{2}{*}{ Conocimiento } & $\begin{array}{l}\text { Autoconomiento: } 24,32 \\
43,55 \text { y } 8\end{array}$ & F1: $11,15,51$ y 20 & Inferior .50: \\
\hline & $\begin{array}{l}\text { Conocimiento de otros: } \\
11,15,20,38,47 \text { y } 51\end{array}$ & F2: $32,55,8$ y 24 & $\begin{array}{l}\text { Complejidad factorial: } 47 \text {, } \\
38 \text { y } 43\end{array}$ \\
\hline Ajuste social & $19,30,42,54,57$ y 44 & $\begin{array}{l}\text { Unifactorial: } 57,64,30 \\
\text { y } 19\end{array}$ & Inferior .60: 42 y 54 \\
\hline Mejora CV & $14,27,35,39$ y 52 & Unifactorial: 39,35 y 27 & Inferior .50: 14 y 52 \\
\hline
\end{tabular}




\begin{tabular}{|c|c|c|c|}
\hline Dimensión & Agrupación teórica & $\begin{array}{l}\text { Agrupación empírica } \\
\text { AFE }\end{array}$ & Ítems eliminados \\
\hline \multirow[t]{4}{*}{ Mejora de Estima } & Estima: $45,28,5,17$ y 53 & $\mathbf{F 1 :} 18,28,17$ y 22 & $\begin{array}{l}\text { Inferior .50: } 62 \text { (.51), 9, 56, } \\
10 \text { y } 5\end{array}$ \\
\hline & $\begin{array}{l}\text { Crecimiento personal: } 62 \text {, } \\
56,48,22 \text { y } 10\end{array}$ & F2: 53,45 y 48 & $\begin{array}{l}\text { Complejidad factorial: } 41 \\
58,9,56 \text { y } 10\end{array}$ \\
\hline & $\begin{array}{l}\text { Relaciones sociales: } 6,9 \text {, } \\
23 \text { y } 58\end{array}$ & F3: 3 y 37 & Carga en factor residual: 6 \\
\hline & Disfrute: $3,18,37$ y 41 & & \\
\hline Condicionales & $4,13,34$ y 59 & $\begin{array}{l}\text { Unifactorial: } 34,59 \\
13 \text { y } 4\end{array}$ & \\
\hline
\end{tabular}

Fuente: elaboración propia

Después de depurar la escala mediante AFE por cada dimensión, se conservaron 39 reactivos. En segundo lugar, se realizó un nuevo Análisis Factorial Exploratorio para estudiar la estructura subyacente de la versión completa de la escala y continuar con el proceso de estudio del instrumento.

Como se observa en la siguiente tabla, los ítems se agruparon en 7 factores y fue posible descartar ciertos reactivos por tener un pesaje inferior a 0,50 y presentar complejidad factorial.

Tabla 9. Ítems conservados e eliminados luego del AFE en escala completa

\begin{tabular}{|c|c|c|}
\hline Factor & Ítems conservados & Ítems eliminados \\
\hline 1 & $44,19,57,59$ y 30 & Inferior 0,50: 13 \\
\hline \multirow[t]{2}{*}{2} & $32,8,45$ y 24 & Inferior 0,50: 17, 22 y 55 \\
\hline & & Complejidad factorial: $28,17,22$ y 55 \\
\hline \multirow[t]{2}{*}{3} & $49,50,60$ y 26 & Inferior 0,50: 48 \\
\hline & & Complejidad factorial: 48 \\
\hline 4 & $11,20,51$ y 15 & Inferior a 0,50: 34 \\
\hline 5 & 35,39 y 14 & Complejidad factorial: 27 y 34 \\
\hline 6 & $1,3,2$ y 12 & Inferior 0,50: 37 \\
\hline \multirow[t]{2}{*}{7} & 25,21 y 36 & Inferior $\mathbf{0 , 5 0 :} 53,7$ y 36 \\
\hline & & Complejidad factorial: 53,36 \\
\hline
\end{tabular}

Fuente: elaboración propia

Finalmente, en tercer lugar, se realizó un último AFE con los 27 ítems seleccionados a partir de los resultados anteriores. La factibilidad del AFE resultó adecuada $\left(K M O=0,90 ; X^{2}\right.$ de Barlett $\left.{ }_{(351)}=4013,40 ; p=0,000\right)$. La matriz factorial quedó 
conformada por siete dimensiones que explican el 56,07\% de la variancia (ver tabla 10 y figura 1).

Tabla 10. Resultados del análisis factorial exploratorio con ítems depurados

\begin{tabular}{|c|c|c|c|c|c|c|c|}
\hline \multirow[b]{2}{*}{ Reactivo } & \multicolumn{7}{|c|}{ Factores } \\
\hline & 1 & 2 & 3 & 4 & 5 & 6 & 7 \\
\hline Ítem 64 & 0,788 & & & & & & \\
\hline Ítem 57 & 0,682 & & & & & & \\
\hline Ítem 59 & 0,667 & & & & & & \\
\hline Ítem 19 & 0,664 & & & & & & \\
\hline Ítem 30 & 0,624 & & & & & & \\
\hline Ítem 49 & & 0,847 & & & & & \\
\hline Ítem 50 & & 0,664 & & & & & \\
\hline Ítem 60 & & 0,630 & & & & & \\
\hline Ítem 26 & & 0,622 & & & & & \\
\hline Ítem 11 & & & 0,755 & & & & \\
\hline Ítem 20 & & & 0,643 & & & & \\
\hline Ítem 15 & & & 0,629 & & & & \\
\hline Ítem 51 & & & 0,613 & & & & \\
\hline Ítem 1 & & & & 0,753 & & & \\
\hline Ítem 2 & & & & 0,585 & & & \\
\hline Ítem 3 & & & & 0,553 & & & \\
\hline Ítem 12 & & & & 0,547 & & & \\
\hline Ítem 32 & & & & & 0,667 & & \\
\hline Ítem 8 & & & & & 0,616 & & \\
\hline Ítem 24 & & & & & 0,535 & & \\
\hline Ítem 45 & & & & & 0,412 & & \\
\hline Ítem 21 & & & & & & 0,781 & \\
\hline Ítem 36 & & & & & & 0,502 & \\
\hline Ítem 25 & & & & & & 0,458 & \\
\hline Ítem 35 & & & & & & & 0,719 \\
\hline Ítem 39 & .518 & & & & & & 0,562 \\
\hline Ítem 14 & & & & & & & 0,357 \\
\hline
\end{tabular}

Nota: factor 1 (ajuste social); factor 2 (conocimiento y comprensión de otros); factor 3 (autoconocimiento y estima propia); factor 4 (reciprocidad); factor 5 (valores vinculados a lo espiritual y al servicio); factor 6 (contribución al desarrollo profesional); factor 7 (transformación social).

Fuente: elaboración propia 


\section{Gráfico de sedimentación}

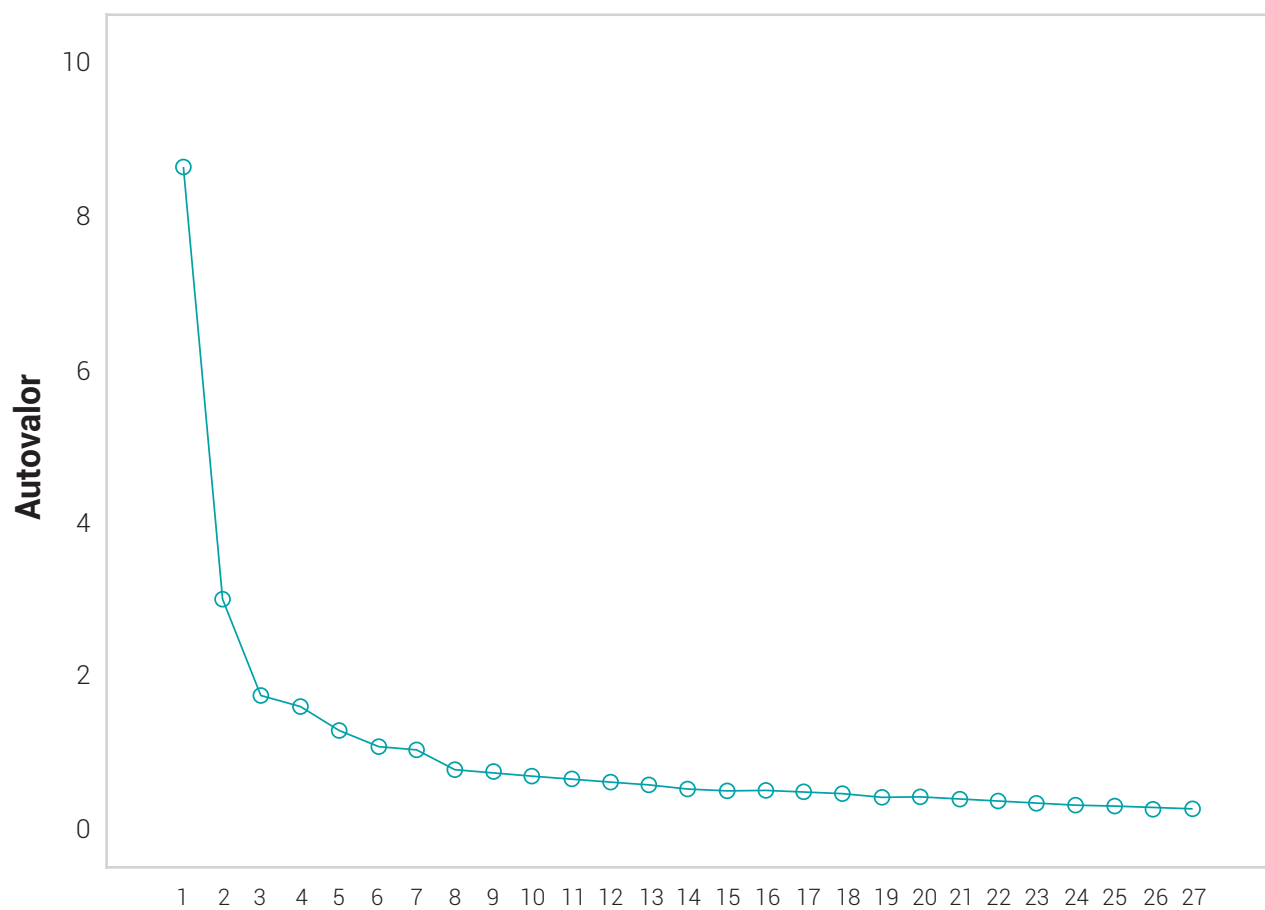

\section{Número de Factor}

Figura 1. Gráfico de sedimentación de los factores que componen el CAMV. Fuente: elaboración propia

Tabla 11. Matriz de correlaciones entre factores

\begin{tabular}{cccccccc}
\hline \multicolumn{7}{c}{ Factores } \\
\hline Factor & $\mathbf{1}$ & $\mathbf{2}$ & $\mathbf{3}$ & $\mathbf{4}$ & $\mathbf{5}$ & $\mathbf{6}$ & $\mathbf{7}$ \\
\hline 1 & 1,000 & $-0,474$ & $-0,376$ & 0,268 & 0,130 & $-0,464$ & $-0,449$ \\
\hline 2 & $-0,474$ & 1,000 & 0,224 & $-0,294$ & $-0,015$ & 0,377 & 0,137 \\
\hline 3 & $-0,376$ & 0,224 & 1,000 & $-0,300$ & $-0,338$ & 0,239 & 0,353 \\
\hline 4 & 0,268 & $-0,294$ & $-0,300$ & 1,000 & 0,216 & $-0,250$ & $-0,307$ \\
\hline 5 & 0,130 & $-0,015$ & $-0,338$ & 0,216 & 1,000 & $-0,061$ & $-0,326$ \\
\hline 6 & $-0,464$ & 0,377 & 0,239 & $-0,250$ & $-0,061$ & 1,000 & 0,308 \\
\hline 7 & $-0,449$ & 0,137 & 0,353 & $-0,307$ & $-0,326$ & 0,308 & 1,000 \\
\hline
\end{tabular}

Nota: factor 1 (ajuste social); factor 2 (conocimiento y comprensión de otros); factor 3 (autoconocimiento y estima propia); factor 4 (reciprocidad); factor 5 (valores vinculados a lo espiritual y al servicio); factor 6 (contribución al desarrollo profesional); factor 7 (transformación social).

Fuente: elaboración propia 


\section{Consistencia interna}

La confiabilidad del instrumento se estudió mediante los coeficientes alpha de Cronbach y el omega de McDonald. En relación al apha de Cronbach se obtuvieron valores de consistencia interna adecuados para la escala en general $(a=0,91)$ y un poco menores para para cada una de las dimensiones. Asimismo, al analizar el coeficiente omega de McDonald se pudieron encontrar valores semejantes para la escala general $(\Omega=0,93)$ y para las dimensiones (ver tabla 12$)$.

Tabla 12. Indicadores de confiabilidad: alpha de Cronbach y omega de McDonald

\begin{tabular}{ccc}
\hline \multicolumn{3}{c}{ Coeficientes } \\
\hline Factor & $\boldsymbol{a}$ & Omega \\
\hline 1 & 0,85 & 0,82 \\
\hline 2 & 0,61 & 0,79 \\
\hline 3 & 0,83 & 0,76 \\
\hline 4 & 0,84 & 0,71 \\
\hline 5 & 0,75 & 0,65 \\
\hline 6 & 0,72 & 0,61 \\
\hline 7 & 0,73 & 0,59
\end{tabular}

Nota: factor 1 (ajuste social); factor 2 (conocimiento y comprensión de otros); factor 3 (autoconocimiento y estima propia); factor 4 (reciprocidad); factor 5 (valores vinculados a lo espiritual y al servicio); factor 6 (contribución al desarrollo profesional); factor 7 (transformación social).

Fuente: elaboración propia

\section{Discusión}

En el campo de la psicología se requiere de herramientas de evaluación que evidencien validez y confiabilidad. De acuerdo a Carretero-Dios y Pérez (2005), la "importación" de instrumentos de evaluación es una práctica frecuente, es decir, se exhiben mayores trabajos dedicados a la adaptación de instrumentos que a la creación de los mismos, a pesar de que en ocasiones las adaptaciones pierden relevancia cultural. La presente investigación tuvo por objetivo el desarrollo de un nuevo instrumento que posibilite evaluar las motivaciones que subyacen en quienes manifiestan interés o deciden involucrarse en actividades de voluntariado.

La teoría sobre la que se construyó el CAMv, parte del enfoque funcional de la psicología social, introducido por Clary et al. (1998), en el campo de las motivaciones para el voluntariado. Los aportes realizados por otros estudios cualitativos y 
cuantitativos en el área también contribuyeron de forma significativa en la presente propuesta de operacionalización del constructo, motivaciones para el voluntariado, destinado a adultos jóvenes (Allison, Okun, y Dutrige, 2002; Chacón y Vecina, 1999; Omoto y Snyder, 1995). Tanto las adaptaciones como las construcciones de instrumentos de evaluación deben realizarse con reflexión, siguiendo las recomendaciones resultantes de estudios que presentan normas para estos procesos (Carretero-Dios y Pérez, 2005).

Los objetivos trazados para la presente investigación se cumplieron. Primero, mediante el desarrollo de las principales consideraciones teóricas respecto al voluntariado y las motivaciones asociadas. Segundo, a través de la presentación del proceso de construcción del CAMV. Durante la construcción de los ítems se tuvo como base los aportes de Chacón et al. (2010), las expresiones utilizadas por voluntarios para describir sus motivaciones y las valoraciones de jueces expertos en evaluación psicológica en cuanto a la pertinencia cultural y correspondencia de los ítems con las categorías de evaluación. El proceso de depuración de ítems del instrumento se realizó mediante el análisis del poder discriminativo y se pudo encontrar que todos los ítems que conformaban inicialmente los grupos de motivaciones resultaron con poder discriminativo. Se estudió también la estructura subyacente de los grupos de motivaciones evaluadas, con la finalidad de continuar con la depuración del instrumento y conocer la agrupación de los ítems en subdimensiones. Los resultados del último AFE realizado a la escala compuesta finalmente por 27 ítems, indicaron una estructura subyacente de siete factores los cuales explicaron un 56,07 \% de la variancia.

Los siete factores hallados fueron los siguientes: 1) Ajuste Social (ítems: 64-5957-19-30), todos los reactivos correspondientes a la categoría Ajuste Social de la escala inicial, excepto el 59 ("Para hacer algo mientras decido qué camino seguir") que pertenecía a la categoría Condicionales. 2) Conocimiento y comprensión de otros (ítems: 11-20-15-51), los cuatro ítems pertenecientes al factor Conocimiento de otros, de la escala inicial. 3) Autoconocimiento y estima propia (32-8-24-45), los reactivos representan a la categoría Autoconocimiento de la escala inicial, excepto el 45 ("Necesidad de sentirme útil") perteneciente a la categoría Estima. 4) Reciprocidad (ítems: 4950-60-26), conformada por reactivos pertenecientes a la categoría Reciprocidad de la escala original. 5) Valores vinculados a lo espiritual y al servicio (ítems: 1-3-2-12), los ítems corresponden a la categoría Valores, excepto el 3 ("Disfruto sirviendo a los demás") que pertenecía a la categoría Disfrute de la escala inicial. 6) Contribución al desarrollo profesional (ítems: 35-39-14), los tres reactivos pertenecían a la dimensión Mejora de CV de la escala inicial. 7) Transformación Social (ítems: 21-25-36), todos los ítems coinciden con la dimensión de Transformación de la versión inicial. 
Con respecto a la confiabilidad de la escala, tanto el coeficiente alpha de Cronbach como el Omega de McDonald indicaron una consistencia interna elevada para la escala general. Los coeficientes por dimensión fueron menores, en la mayoría, adecuados, aunque algunos un poco por debajo de los límites recomendados. Es esperable que al reducir el número de ítems incluidos en el análisis se reduzca el coeficiente de confiabilidad, ya que el grado de correlación es muy sensible al $n$ (Cronbach, 1951). Igualmente, se recomienda, para un siguiente estudio, revisar dichas dimensiones ajustando o incluyendo nuevos reactivos a fin de elevar la consistencia interna de las mismas.

Contar con una herramienta como el CAMV, que permita evaluar el constructo motivaciones para el voluntariado en adultos jóvenes argentinos, puede contribuir a las estrategias de promoción del voluntariado, teniendo en cuenta que gran parte de las necesidades que se demandan en contextos sociales dependen del trabajo de voluntarios (Hustinx, et al., 2010). Asimismo, podría ser útil en la selección de voluntarios y asignación de tareas. Contemplar el aspecto de las motivaciones de los postulantes a voluntarios, podría repercutir en la calidad de su trabajo y permanencia en el puesto.

Dada la extensión del presente artículo, se propone para una próxima investigación, el análisis de otras evidencias de validez externa y la confirmación de la estructura observada en el instrumento desarrollado, a partir de un Análisis Factorial Confirmatorio.

\section{Referencias}

Allison, L. D., Okun, M. A. and Dutridge, K. S. (2002). Assessing volunteer motives: a comparison of an open-ended probe and Likert rating scales. Journal of Community \& Applied Social Psychology, 12(4), 243-255. doi:10.1002/casp.677

Bang, H. and Ross, S. D. (2009). Volunteer motivation and satisfaction. Journal of Venue and Event Management, 1(1), 61-77.

Batson, C. D. (1991). The altruism question: Toward a social- psychological answer. Hillsdale, NJ: Lawrence Erlbaum Associates Publishers. doi: 10.4324/9781315808048

Batson, C. D. and Ahmad, N. (2001). Empathy-induced altruism in a prisoner's dilemma II: What if the target of empathy has defected. European Journal of Social Psychology, 31(1), 25-36. 
Burns, D. J., Toncar, M., Reid, J., Anderson, C., Wells, C., Fawcett, J. and Gruben, K. (2005). Volunteering: A comparison of the motivations of collegiate students attending different types of institutions. Journal of Volunteer Administration, 23(4), 31.

Butt, M. U., Hou, Y., Soomro, K. A. and Acquadro Maran, D. (2017). The ABCE Model of Volunteer Motivation. Journal of Social Service Research, 43(5), 593-608. doi: 10.1080/01488 376.2017 .1355867

Carlo, G., Okun, M. A., Knight, G. P. and de Guzman, M. R. T. (2005). The interplay of traits and motives on volunteering: Agreeableness, extraversion and prosocial value motivation. Personality and Individual Differences, 38(6), 1293-1305. doi: 10.1016/j.paid.2004.08.012

Carretero-Dios, H., y Pérez, C. (2005). Normas para el desarrollo y revisión de estudios instrumentales. International Journal of clinical and health psychology, 5(3), 521-551.

Chacón, F. y Vecina, M. L. (1999). Análisis de la fiabilidad de un cuestionario de motivaciones del voluntariado en SIDA. Psicothema, 11(1), 211-223. doi: 10.1174/02109390260288613

Chacón, F., Pérez, T., Flores, J. y Vecina, M. L. (2010). Motivos del voluntariado: categorización de las motivaciones de los voluntarios mediante pregunta abierta. Psychosocial Intervention, 19(3), 213-222. doi: 10.5093/in2010v19n3a2

Clary, E. G. and Snyder, M. (1991). A functional analysis of altruism and prosocial behavior: The case of volunteerism. Review of Personality and Social Psychology, 12, 119-148.

Clary, E. G. and Snyder, M. (1999). The motivations to volunteer: Theoretical and practical considerations. Current directions in psychological science, 8(5), 156-159.

Clary, E. G., Snyder, M., Ridge, R. D., Copeland, J., Stukas, A. A., Haugen, J., y Miene, P. (1998). Understanding and assessing the motivations of volunteers: a functional approach. Journal of personality and social psychology, 74(6), 1516. doi: 10.1037/0022-3514.74.6.1516

Cnaan, R. A. and Goldberg-Glen, R. S. (1999). Measuring motivation to volunteer in human services. Journal of Composite Materials, 33(10), 928-940.

Cronbach, L. J. (1951). Coefficient alpha and the internal structure of tests. Psychometrika, 16(3), 297-334.

Curtis, J. E., Grabb, E. G. and Baer, D. E. (1992). Voluntary association membership in fifteen countries: A comparative analysis. American Sociological Review, 139-152. doi: 10.2307/2096201 
Dutta-Bergman, M. J. (2004). Describing volunteerism: The theory of unified responsibility. Journal of Public Relations Research, 16(4), 353-369. doi: 10.1207/s1532754xjprr1604_2

Eisenberg, N. and Fabes, R. A. (1990). Empathy: Conceptualization, measurement, and relation to prosocial behavior. Motivation and Emotion, 14(2), 131-149. doi: 10.1007/bf00991640

Erasmus, B. and Morey, P. J. (2016). Faith-based volunteer motivation: Exploring the applicability of the volunteer functions inventory to the motivations and satisfaction levels of volunteers in an Australian faith-based organization. Voluntas, 27(3), 1343-1360. doi: 10.1007/ s11266- 016-9717-0

Erasmus, B. and Morey, P. J. (2016). Faith-based volunteer motivation: Exploring the applicability of the volunteer functions inventory to the motivations and satisfaction levels of volunteers in an Australian faith-based organization. Voluntas: International Journal of Voluntary and Nonprofit Organizations, 27(3), 1343-1360.

Fitch, T. (1987). Characteristics and motivations of college students volunteering for community service. Journal of College Students Personnel, 28(5), 424-431.

Frisch, M. B. and Gerrard, M. (1981). Natural helping systems: A survey of Red Cross volunteers. American Journal of Community Psychology, 9(5), 567-579. doi: 10.1007/ BF00896477

Gage, R. L. and Thapa, B. (2012). Volunteer motivations and constraints among college students: Analysis of the volunteer function inventory and leisure constraints models. Nonprofit and Voluntary Sector Quarterly, 41(3), 405-430.

Garipova, A. N. (2016). Volonteering motivation in student's life. In Modern look at the future of science collection: Articles of the International scientific-practical conference.-Tomsk (pp. 7880). (s.d.)

Geiser, C., Okun, M. A. and Grano, C. (2014). Who is motivated to volunteer? A latent profile analysis linking volunteer motivation to frequency of volunteering. Psychological Test and Assessment Modeling, 56(1), 3-24.

Gibbons, P., Flores de Arévalo, H. and Mónico, M. (2004). Assessment of the factor structure and reliability of the 28-item version of the General Health Questionnaire (GHQ-28) in El Salvador. International journal of clinical and health psychology, 4(2), 389-398.

Gidron, B. (1985). Predictors of retention and turnover among service volunteer workers. Journal of Social Service Research, 8(1), 1-16. doi: 10.1300/j079v08n01_01 
Hallmann, K. and Harms, G. (2012). Determinants of volunteer motivation and their impact on futurevoluntary engagement: A comparison of volunteer motivation at sport events in equestrian and handball. International Journal of Event and Festival Management, 3(3), 272-291. doi: $10.1108 / 17582951211262701$

Hettman, D. W. and Jenkins, E. (1990). Volunteerism and social interest. Individual Psychology, 46(3), 298-303.

Hibbert, S., Piacentini, M. and Dajani, H. A. (2003). Understanding volunteer motivation for participation in a community-based food cooperative. International Journal of Nonprofit and Voluntary Sector Marketing, 8(1), 30-42. doi: 10.1002/nvsm.199

Hustinx, L., Handy, F., Cnaan, R. A., Brudney, J. L., Pessi, A. B. and Yamauchi, N. (2010). Social and cultural origins of motivations to volunteer: A comparison of university students in six countries. International Sociology, 25(3), 349-382. doi: 10.1177/0268580909360297

Jiranek, P., Kals, E., Humm, J. S., Strubel, I. T. and Wehner, T. (2013). Volunteering as a means to an equal end? The impact of a social justice function on intention to volunteer. The Journal of Social Psychology, 153(5), 520-541. doi: 10.1080/00224545.2013.768594

Latting, J. K. (1990). Motivational differences between Black and White volunteers. Nonprofit and Voluntary Sector Quarterly, 19(2), 121-136. doi: 10.1177/089976409001900204

Lloret-Segura, S., Ferreres-Traver, A., Hernández-Baeza, A. y Tomás-Marco, I. (2014). El análisis factorial exploratorio de los ítems: una guía práctica, revisada y actualizada. Anales de psicología, 30(3), 1151-1169. doi: 10.6018/analesps.30.3.199361

Montero, I. y León, O. G. (2005). Sistema de clasificación del método en los informes de investigación en Psicología. International Journal of clinical and health psychology, 5(1), 115-127.

Omoto, A. M. and Snyder, M. (1995). Sustained helping without obligation: motivation, longevity of service, and perceived attitude change among AIDS volunteers. Journal of personality and social psychology, 68(4), 671-686. doi: 10.1037/0022-3514.68.4.671

Parboteeah, K. P., Cullen, J. B. and Lim, L. (2004). Formal volunteering: A cross-national test. Journal of World Business, 39(4), 431-441. doi: 10.1016/j.jwb.2004.08.007

Penner, L. A. (2002). Dispositional and organizational influences on sustained volunteerism: An interactionist perspective. Journal of Social Issues, 58(3), 447-467. doi: 10.1111/1540-4560.00270 
Penner, L. A. and Finkelstein, M. A. (1998). Dispositional and structural determinants of volunteerism. Journal of Personality and Social Psychology, 74(2), 525-537. doi: 10.1037/ 0022-3514.74.2.525

Penner, L. A., Midili, A. R. and Kegelmeyer, J. (1997). Beyond job attitudes: A personality and social psychology perspective on the causes of organizational citizenship behavior. Human Performance, 10(2), 111-132. doi: 10.1207/s15327043hup1002_4

Pham, T. (2019). Volunteer Motivation and Retention in a Nonprofit Organization. Recuperado de: https://hdl.handle.net/11244/319773

Rokach, A. and Wanklyn, S. (2009). Motivation to volunteer: Helping empower sick children. Psychology and Education Journal, 46(1), 7-25.

Servera, M. and Cardo, E. (2006). Children sustained attention task (CSAT): Normative, reliability, and validity data. International Journal of Clinical and Health Psychology, 6(3), 697-707.

Shye, S. (2010). The Motivation to Volunteer: A Systemic Quality of Life Theory. Social Indicators Research, 98(2), 183-200.

Smith, D. H. (1981). Altruism, volunteers, and volunteerism. Journal of Voluntary Action Research, 10(1), 21-36.

Smith, J. (2009). Solidarity networks: what are they? And why should we care? The Learning Organization, 16(6), 460-468.

Stukas, A. A., Daly, M. and Clary, G. E. (2006). Lessons from research on volunteering for mobilizing adults to volunteer for positive youth development. In E. G. Clary and J. E. Rhodes. (Eds.), Mobilizing adults for positive youth development (pp. 65-82). New York, USA: Springer. doi: 10.1007/0-387-29340-X_4

Stukas, A. A., Hoye, R., Nicholson, M., Brown, K. M. and Aisbett, L. (2016). Motivations to volunteer and their associations with volunteers' well-being. Nonprofit and Voluntary Sector Quarterly, 45(1), 112-132.

Thoits, P. A. and Hewitt, L. N. (2001). Volunteer work and well-being. Journal of health and social behavior, 42(2), 115-131.

Tornimbeni, S., Pérez, E. y Olaz, F. (2008). Introducción a la psicometría. Buenos Aires: Paidós. 
United Nations Volunteers (2011). V Informe sobre el estado del voluntariado en el mundo. Dinamarca: Phoenix Design Aid.

Van Vianen, A. E. M., Nijstad, B. A. and Voskuijl, O. F. (2008). A person-environment fit approach to volunteerism: Volunteer personality fit and culture fit as predictors of affective outcomes. Basic and Applied Social Psychology, 30(2), 153-166. doi: 10.1080/01973530802209194

Wilson, J. and Musick, M. (1997). Who cares? Toward an integrated theory of volunteer work. American Sociological Review, 62(5), 694-713. doi: 10.2307/2657355

\section{Anexo}

Cuestionario argentino de motivaciones para el voluntariado (ítems)

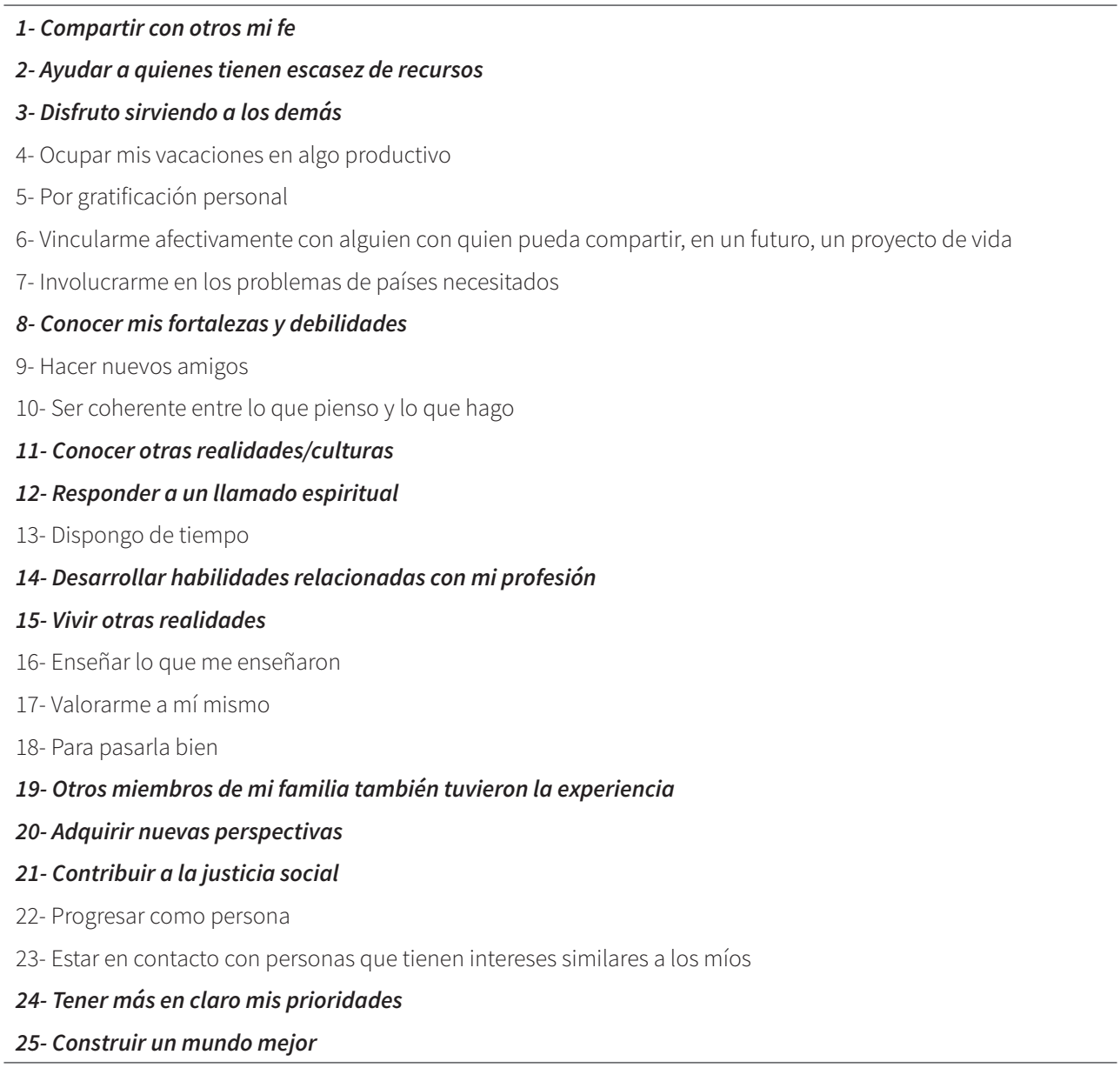


(viene)

\author{
26- Ayudar como antes me ayudaron a mí \\ 27- Podría incidir en mis posibilidades de inserción laboral \\ 28- Para sentirme bien conmigo mismo \\ 29- Compartir mis creencias religiosas con otros
}

30- Mi entorno considera importante que tenga estas experiencias

31- Hacer algo por mi comunidad

32- Conocerme mejor a mi mismo

33- Aportar mi grano de arena para mejorar el mundo

34- Para hacer algo diferente

35- Adquirir experiencia profesional

36- Disminuir aquello que considero injusto

37- Es algo que me gusta

38- Sentimientos de curiosidad por las vivencias de otros

39-Aportar a mi currículum y a mi desarrollo profesional

40- Asumir mi responsabilidad religiosa

41- Ocupar mi tiempo en algo que pueda disfrutar

42- Personas que admiro realizaron actividades de voluntariado

43- Darme cuenta de todo lo que tengo

44- Por recomendación/estimulación de mis padres o familiares

45- Necesidad de sentirme útil

46- Colaborar con alguna comunidad que esté pasando por una dificultad

47- Aprender en situaciones nuevas

48- Desarrollar al máximo mis potencialidades

49- Devolver un poco de lo que a mí me dieron

50- Expresar sentimientos de gratitud

51- Aprender a adaptarme a distintas situaciones/culturas

52- Descubrir mi vocación

53- Darme cuenta que puedo hacer algo por otros

54- Compartir momentos con amigos que también se involucran

55- Profundizar en mi mundo interno

56- Ser consecuente con mis valores

57- Estimulación por parte de mis docentes

58- Conocer personas con las mismas inquietudes

59- Para hacer algo mientras decido qué camino seguir

60- Compartir lo que he recibido

61- Ayudar a personas excluidas

62- Enriquecerme o crecer personalmente 